\section{L'AMP-activated protein kinase hypothalamique, régulateur essentiel du poids et de la prise alimentaire}

Fabrizio Andreelli
Service de Diabétologieendocrinologie-nutrition, Hôpital Bichat-Claude

Bernard,

16, rue Henri Huchard,

75877 Paris Cedex 18,

France.

fabrizio.andreelli@

bch.ap-hop-paris.fr

un acide gras particulier, à chaîne courte, cofacteur essentiel des enzymes de la respiration mitochondriale (Figure 1) [2]. L' $\alpha$-LA réduit la prise alimentaire chez le rat Sprague-Dawley quelle que soit sa voie d'administration (orale, intrapéritonéale ou intracérébro-ventriculaire). Cet effet est dépendant de la dose. L' $\alpha$ $\mathrm{LA}$ réduit le poids et la masse grasse intraviscérale des rats génétiquement obèses (rats Otsuka, Long-Evans, Tokushima, Fatty ou rats OLETF) et améliore leurs paramètres métaboliques. L'effet anorexigène du $\alpha$-LA est réversible à l'arrêt de son administration, suggérant que cet effet n'est pas dépendant d'un éventuel effet toxique de l'acide gras. De plus, l' $\alpha$-LA augmente la dépense énergétique (mesurée par calorimétrie indirecte) et augmente l'expression de la protéine découplante UCP-1 dans le tissu adipeux brun. Ainsi, I' $\alpha$-LA semble réduire le poids en diminuant la prise alimentaire mais également en stimulant la dépense calorique journalière. Ces effets, mêmes s'ils apparaissent similaires à ceux observés après l'administration de leptine, sont indépendants de la voie de signalisation de la leptine. $\varepsilon$ n effet, l' $\alpha$-LA conserve son action chez les souris obèses déficientes en leptine ou en son récepteur. De plus, à l'inverse de ce qui est observé pour la leptine, l'administration intrapéritonéale d' $\alpha$ - LA ne s'accompagne pas d'une diminution d'expression hypothalamique du neuropeptide Y (NPY), de la pro-opiomélanocortine (POMC) ou de la corticotropin releasing hormone (CRH). Quel est donc 
le médiateur hypothalamique des effets de I' $\alpha$-LA? L'injection intrapéritonéale d' $\alpha$-LA réduit l'activité de l'AMPK hypothalamique et plus particulièrement l'activité relayée par la sous-unité catalytique $\alpha 2$ dans le noyau arqué sans affecter I'AMPK- $\alpha 2$ du noyau paraventriculaire ou de l'aire hypothalamique latérale. Les effets pondéraux de l' $\alpha$-LA sont bien secondaires à une réduction de l'activité de l'AMPK- $\alpha 2$ puisque les effets de l' $\alpha$-LA sur la prise alimentaire sont abolis lors de la co-injection intracérébro-ventriculaire d' $\alpha-L A$ et d'un activateur de I'AMPK (dénommé AICAR) ou lors de l'injection intrapéritonéale d' $\alpha$-LA chez des rats exprimant dans I'hypothalamus une forme constitutivement active d'AMPK.

L'activité de l'AMPK- $\alpha 2$ hypothalamique est contrôlée par l'acide gras $\alpha$-LA. Qu'en est-il pour le glucose, autre substrat énergétique majeur? L'injection intracérébro-ventriculaire de glucose (5 mg) chez des rats à jeun depuis 24 heures réduit l'activité de l'AMPK- $\alpha 2$. À l'inverse, l'injection intrapéritonéale de 2-désoxyglucose (qui inhibe l'utilisa- tion du glucose intracellulaire, mimant ainsi une carence en glucose) chez des rats nourris augmente malgré tout leur prise alimentaire via une augmentation de l'activité de l'AMPK- $\alpha 2$ hypothalamique. L'effet du 2-désoxyglucose n'était plus retrouvé chez des rats exprimant un dominant négatif de I'AMPK dans l'hypothalamus.

L'ensemble de ces données montre que I'AMPK hypothalamique participe à la régulation de la prise alimentaire et de la dépense énergétique et que des substrats aussi divers que certains acides gras (comme l' $\alpha$-LA) et le glucose modulent l'activité de cette enzyme. Globalement, I' $\alpha$-LA et le glucose réduisent l'activité de l'AMPK- $\alpha 2$ hypothalamique alors que la carence en glucose active l'enzyme, confirmant que I'AMPK$\alpha 2$ est bien un régulateur énergétique à l'échelon de l'hypothalamus. Les conséquences des modifications d'activité de I'AMPK- $\alpha 2$ sont considérables, touchant la prise alimentaire et la dépense énergétique (même si les mécanismes exacts de leur régulation sont encore inconnus). De plus, contrairement à l'article publié par l'équipe de B.B. Kahn [3] quelques mois auparavant, et qui suggérait que les effets centraux de la leptine sont relayés par I'AMPK, l'article de M.S. Kim et al. démontre clairement que la régulation de I'AMPK hypothalamique est indépendante des principaux régulateurs hypothalamiques connus de la machinerie énergétique et de la prise alimentaire (leptine, POMC, CRH, NPY), suggérant que l'inhibition de l'AMPK pourrait être une nouvelle voie thérapeutique dans la prise en charge de l'obésité. $\diamond$

Hypothalamic AMP-activated protein kinase, a regulator of body weight and food intake

\section{RÉFÉRENCES}

1. Kim MS, Park JY, Namkoong C, et al. Anti-obesity effects of $\alpha$-lipoic acid mediated by suppression of hypothalamic AMP-activated protein kinase. Nat Med 2004; 10: 727-33.

2. Viollet B, Andreelli F, Jorgensen SB, et al. The AMPactivated protein kinase alpha2 catalytic subunit controls whole-body insulin sensitivity. J Clin Invest 2003; 111: 91-8.

3. Minokoshi Y, Alquier T, Furukawa N, et al. AMP-kinase regulates food intake by responding to hormonal and nutrient signals in the hypothalamus. Nature 2004; 428: $569-74$

\section{NOUVELLE}

\section{Les exosomes:}

\section{des convoyeurs de prions?}

Benoît Février, Hubert Laude, Graça Raposo, Didier Vilette
> Peu étudiées jusqu'au récent épisode de l'encéphalopathie spongiforme bovine, les maladies à prions - ou encore encéphalopathies spongiformes transmissibles sont des maladies neurodégénératives à l'issue invariablement fatale qui touchent aussi bien l'homme (maladie de Creutzfeldt-Jakob) que l'animal [1]. La conversion, dans les tissus infectés, d'une protéine cellulaire, la PrP, en une isoforme conformationnelle anormale et pathologique est un événement central de ces affections. La PrP anormale (ou PrPSc) serait non seulement à l'origine de la neurodégénérescence [2] et des dysfonctionnements neurologiques observés dans ces maladies mais aussi, selon l'hypothèse dite «de la protéine seule», l'agent infectieux [3]. La conversion de la PrP en PrPSc, via une interaction des deux isoformes, constituerait ainsi le mode de multiplica- tion de ces agents infectieux non conventionnels.

Si les lésions dégénératives observées en phase terminale sont restreintes au système nerveux central, ce tissu ne constitue pas pour autant le siège exclusif de la multiplication des prions. Dans la grande majorité des cas, les contaminations ont en effet lieu par voie périphérique, le plus souvent par voie orale. Après franchissement de la barrière intestinale, l'agent infectieux se multiplie dans diverses formations lymphoïdes (ganglions, rate...), puis colonise le système nerveux central via les trajets nerveux 